\title{
Marijuana Usage, the Effects in Oral Health
}

\author{
Ilma Robo ${ }^{1}$, Oresto Çuni ${ }^{1}$, Ermelinda Gina ${ }^{2}$, Saimir Heta $^{3}$, Luan Mavriqi ${ }^{1}$ \\ ${ }^{1}$ Department of Stomatology, Faculty of Medical Sciences, Albanian University, Tirana, Albania \\ ${ }^{2}$ Private Dental Clinic, Tirana, Albania \\ ${ }^{3}$ University Hospital Center, Pediatric Surgery, Tirana, Albania
}

Email address:

ilmarobo@yahoo.com (I. Robo)

\section{To cite this article:}

Ilma Robo, Oresto Çuni, Ermelinda Gina, Saimir Heta, Luan Mavriqi. Marijuana Usage, the Effects in Oral Health. International Journal of Dental Medicine. Vol. 4, No. 1, 2018, pp. 1-4. doi: 10.11648/j.ijdm.20180401.11

Received: December 27, 2017; Accepted: February 9, 2018; Published: March 6, 2018

\begin{abstract}
Oral health workers should be alert about the dangers of marijuana use in the screening of oral pathologies and methods of administration of similar clinical cases. The use of marijuana is for medicinal and recreational reasons. It is already known, and based on the literature, this plant causes besides medicinal advantages, oral health pathologies also. For carrying out the study, was committed a group of students, who examined clinically, 3 patients, that later were diagnosed. Reduced saliva, influenced by use of marijuana, encourages the show of xerostomia. In this way, this plant is a favorable factor for caries that at examined patients was currently, more than at 5 teeth. Dysplastic changes in the epithelium of the buccal mucosa were appearing as the result of the action of chemical factors of marijuana smoke. Inflammation of the gums, gingivitis sometimes called "fiery red", is the lesion that causes great pain. The use of anesthesia with epinephrine during dental procedures, to users of marijuana may cause acute anxiety or tachycardia. Candinda Albicans was promoted as the result of the presence of carbon hydroxide at the smoke of marijuana. Chronic marijuana usage causes pathologies mentioned above, at individuals who do not treat in time. Counseling and education of oral health workers and individual users or not, about these pathologies, represents the largest contribution in diagnostic routine oral work.
\end{abstract}

Keywords: Marijuana, Oral Health, Pathology, Awareness, Chronic Use

\section{Introduction}

The use of marijuana is done for medical and recreational reasons therefore, in addition to the medical benefits, it also causes oral health pathology. Periodontal literature has limited references to the periodontal effect of cannabis use [1].

About 8.1 million people in the U.S.A are daily or nearly daily users, according to a report of the National Survey on Drug Use and Health of 2015 [2]. Both tobacco and marijuana smoke contain high concentrations of many substances, some of which are recognized by science as carcinogenic substances. These chemical substances put marijuana users at risk for many oral cavity problems, such as periodontal disease, dry mouth (xerostomia), dental caries, and even oral cancer [3]. According to this study, high susceptibility to peridontal disease is reported in marijuana users, a study conducted in the sample of 1,015 people with a mean age of 32 years. The long-term effects of long-term use of marijuana are not well-documented and the state of availing status is still good. Marijuana distinction since marijuana cigarettes do not have the filter part of cigarettes. [3] In this way, marijuana users can inhale deeper the vapor and smoke to keep it longer in the lungs. This may be the reason for promoting lung cancer because the remains in them are at higher levels. With the legalization of marijuana [4], the number of users in different countries increases, so the number of individuals affected simultaneously by the oral and pulmonary tumors, both simultaneously appeared. Cannabis smoking may be a risk factor for periodontal disease that is independent of the use of tobacco [3]. The full extent of long-term health risks of chronic cannabis use (if today's young smokers continue the habit) may require a latent period of 10-20 years to be revealed [5]. Oral health workers should be alert about the dangers of marijuana use in the screening of oral pathologies and methods of administration of similar clinical cases. The use of marijuana is for medicinal and recreational reasons. It is already known, 
and based on the literature, this plant causes besides medicinal advantages, oral health pathologies also. Reduced saliva, influenced by use of marijuana, encourages the show of xerostomia. In this way, this plant is a favorable factor for caries that at examined patients was currently, more than at 5 teeth. Dysplastic changes in the epithelium of the buccal mucosa were appearing as the result of the action of chemical factors of marijuana smoke. Inflammation of the gums, gingivitis sometimes called "fiery red", is the lesion that causes great pain. The use of anesthesia with epinephrine during dental procedures, to users of marijuana may cause acute anxiety or tachycardia. Candinda Albicans was promoted as the result of the presence of carbon hydroxide at the smoke of marijuana. Chronic marijuana usage causes pathologies mentioned above, at individuals who do not treat in time. Counseling and education of oral health workers and individual users or not, about these pathologies, represents the largest contribution in diagnostic routine oral work.

\section{Method}

Patient R.H at age 23 (figure 1), after the examination was diagnosed with: periodontal problems, inflammatory gingiva, alba line highlighted as the result of stressful condition. The gingival hypertrophy caused by marijuana is susceptible to hypertrophy caused by phenytoin [7].
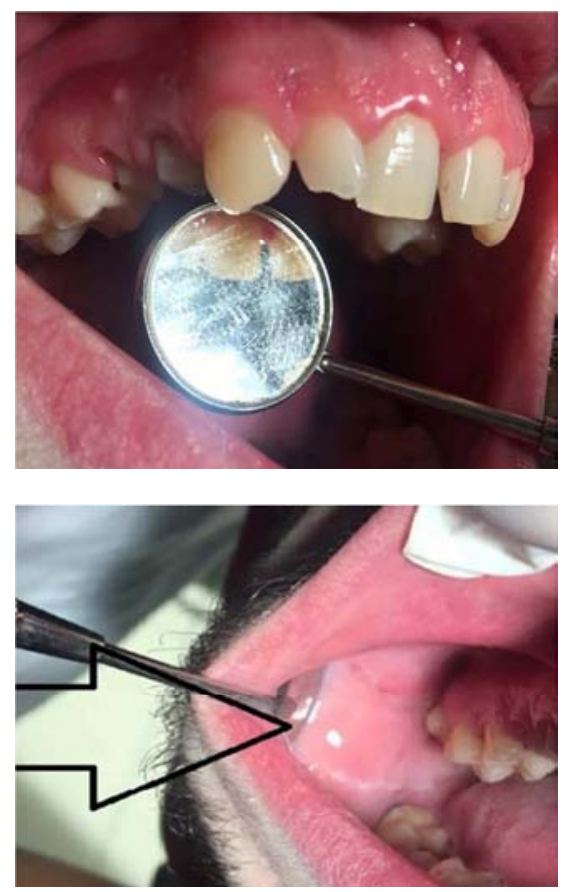

Figure 1. Gingival hypertrophy in the margins of the gingiva and alba line in the inner part of the page is distinguished in the figure.

The correlation between the use of marijuana and the risk of oral cancer appears to be assessed, especially after assessing the level of alcohol use and smoking [8]. Figure 2 shows the same patient in Figure 1, but it is better to distinguish the inflammatory hypertrophy caused by the presence of peridontal bacterial plaque at 21 and the use of marijuana at 11 hypertrophy.
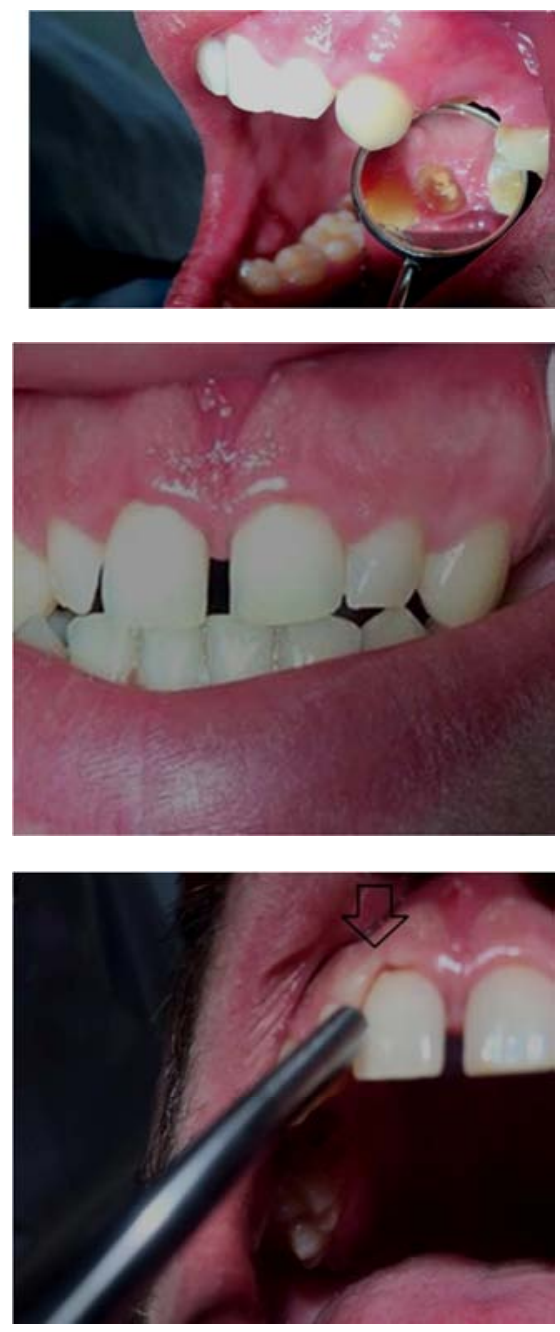

Figure 2. The same patient is demonstrated in this figure, where the difference in gingival inflammation caused by the presence of the periodontal stone at tooth no. 21 and gingival hypertrophy caused by marijuana to tooth no.11.

Patient A.N, at age 20 years, after the examination was diagnosed with: class $\mathrm{V}$ caries on all maxilla fronts, with periodontal problems, inflammatory gingiva, xerostomia, white lesions in the periodontal structures (figure 3 ).

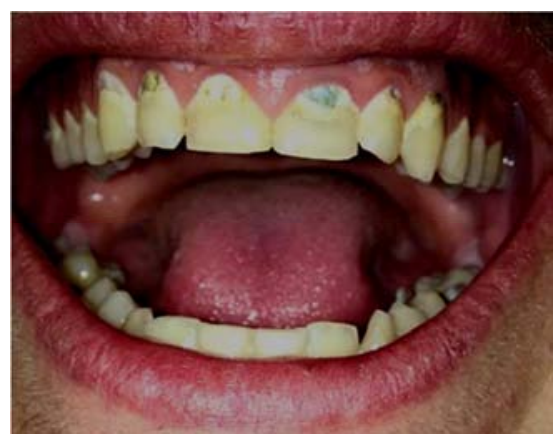




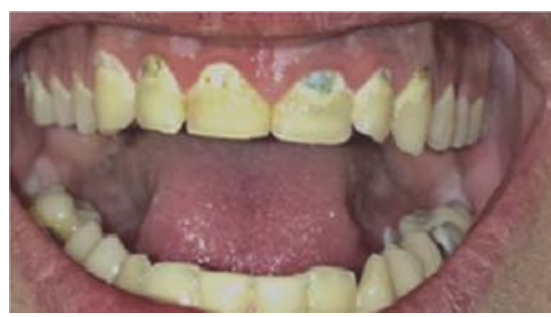

Figure 3. The patient was presented to the clinic, with fifth class lesson according to Black.

Patient M.B. at age 23 years after the examination was diagnosed with: Caries in all molars down and over, caries in fronts above 11, 22, dry mouth (xerostomia), gingival inflammation (figure 4).

After the dental procedure, the patient appeared with dysphoria, acute anxiety and tachycardia. After examination by the appropriate doctor, arrive to the conclusion that these symptoms were caused by epinephrine in anesthesia used for dental procedures.
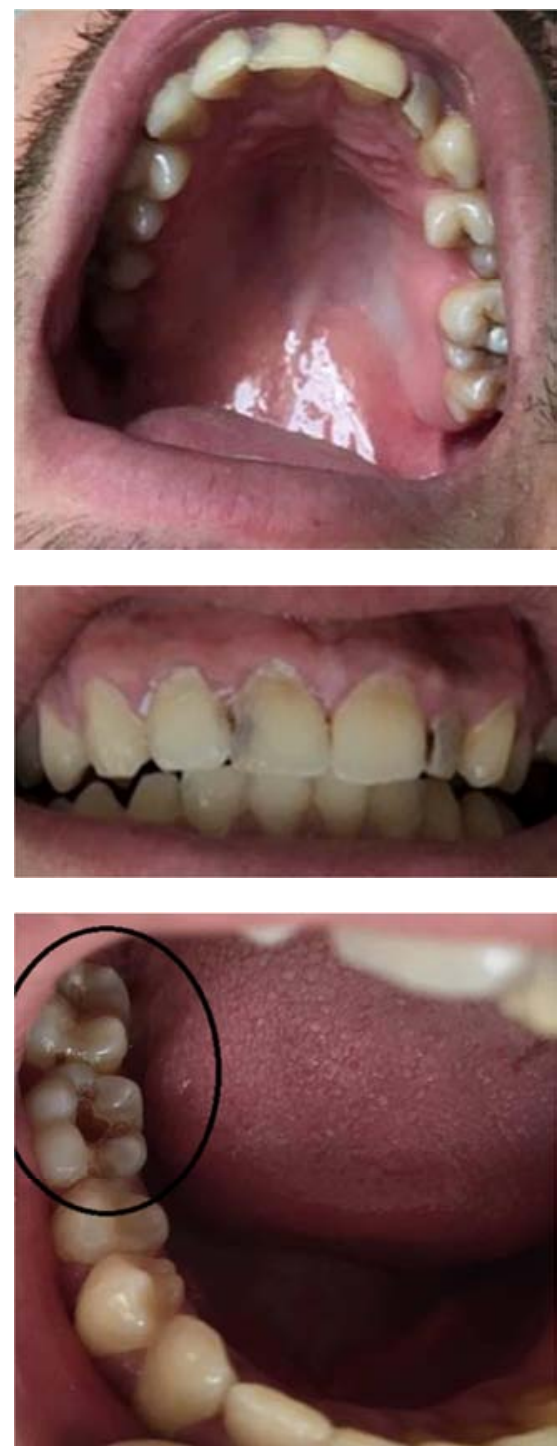

Figure 4. The figure shows the patient with caries of different classes. The patient is a drug user for 5 years.

\section{Results}

The mechanism that marijuana acts as a carcinogen is related to the presence of aromatic carbon hydroxyl, benzopyridine and nitro-amine associated with dysplasic changes in the buccal mucus epithelium [9]. Gingivitis called "fiery red" that causes pain, is noticed in user patients, as well as occasionally loss of alveolar bone.

In case of doubt about the use of marijuana by the patient and to make it easier for a proper examination, the dentist should make the patient feel comfortable while the patient is in the dental chair.

The first thing to note is that there is no marijuana stereotype, that cannot qualify as drugs consumed by the poor or the rich, no culture or age, so as far as it is concerned, as professionals should have tone of voice the smoother and more social with the patient, to achieve the cooperation. As dental professionals, it is timely to incorporate queries about patients' patterns of cannabis use as part of the medical history, just as enquiries about tobacco smoking have been added to the medical history in recent times [10].

Attempting to sensitize as few patients as not to use and starting the procedures of a clean oral hygiene would be plus for the dentist. The chronic use of marijuana may lead to suppression in the immune regulatory system and reduce the body's ability to produce antibodies [11].

\section{Discussion}

Substantial availability and usage of cannabis, specifically among young adults, requires dentists to be vigilant about clinical indications of cannabis use and to provide appropriate treatments. Behavioral modification, nonsurgical therapy, and surgical therapy offer the potential for successful management of cannabis-related periodontitis [12]. The treatment of chronic pain with medicinal cannabis in this open-label, prospective cohort resulted in improved pain and functional outcomes, and a significant reduction in opioid use. Results suggest long-term benefit of cannabis treatment in this group of patients, but the study's noncontrolled nature should be considered when extrapolating the results [13]. Oral health workers should be alert about the dangers of marijuana use in the screening of oral pathologies and methods of administration of similar clinical cases. The use of marijuana is for medicinal and recreational reasons. It is already known, and based on the literature, this plant causes besides medicinal advantages, oral health pathologies also.

Presentation of the patient in the dental clinic is also the first presentation with bodily characteristics. The eye is taught to estimate the most sensitive proportion of body length and body weight. This report thinks that both smokers and those who use marijuana are in the direction of reducing body weight in relation to the rate. Central effects of cannabinoids include disruption of psychomotor behavior, short-term memory impairment, intoxication, stimulation of appetite, antinociceptive actions (particularly against pain of neuropathic origin) and anti-emetic effects [6]. The purpose 
of this study is to sensitize the non-use of narcotic substances, with the follow-up and treatment of various oral pathologies caused by the use of marijuana. During the clinical examination of the oral cavity and during the periodontal status registration, one can clearly distinguish a smoker from a marijuana user. Even lesions caused by both substances, especially those with tumor can be diagnosed and then treated depending on which of the substances the patient uses. Normally, we expect that during the examination of smokers bleeding gingiva will appear, high amount of bacterial plaque and possibly painted with characteristic pigments of nicotine, and in fact, the smoking patient appears with regular oral hygiene, may suffer from coloration of the surface, and with minimum oral plaque. Based on studies, it is noted that smoking is associated with less bleeding and less gingival pockets depths, compared with non-smoker patients [3]. Based on the relevant literature, a group of students led by the dentist was engaged in the realization of this study. Clinically examined, some patients, users, and then were diagnosed by the dentist.

\section{Conclusions}

The use of marijuana reduces the amount of saliva produced in the mouth, due to its $\mathrm{SN}$ effects. With frequently use this effect results in an uncomfortable condition, called dry mouth or xerostomia. Without enough saliva, foods and bacteria become a problem for oral hygiene. Chrysostomy can cause bad breath and wound in the mouth.

In addition, dry mouth encourages tooth decay (favoring caries factor) and their possible loss if failure is not treated in time. Also, Candida Albicans may be present in consumer patients (due to the presence of carbon hydroxide in plant smoke). Users have poor oral hygiene that increases the likelihood of exposure to caries and periodontal disease.

Careful! Patients during or after the procedures may experience acute anxiety, dysmorphia and tachycardia due to epinephrine in anesthesia. So, anesthesia without epinephrine is recommended.

\section{References}

[1] Rawal SY, Tatakis DN, Tipton DA; "Periodontal; and oral manifestations of marijuana use"; J Tenn Dent Assoc; 2012 Fal-Winter; 92(2):26-31; quiz 31-2.

[2] NASADAD•1025 Connecticut AveNW, Ste.605 • Washington, DC 20036 • T: (202) 293-0090・ F: (202) 293-1250 - Website: www.nasadad.org.

[3] D. K. Gautam, V. Jindal, S. C. Gupta, A.Tuli, B.Kotwal, and R. Thakur, "Effect of cigarette smoking on the periodontal health status: A comparative, cross sectional study," J. Indian Soc. Periodontol., vol. 15, no. 4, pp. 383-387, Oct. 2011. DOI: 10.4103/0972-124X.92575; PMid: 22368364; PMCid: PMC3283937.

[4] Iversen L. (The science of Marijuana) USA: oxford university press, 2000.

[5] Ashton CH. Pharmacology and effect of cannabis: a brief review. Br J psychiatry 2001

[6] Iversen L. (Cannabis and the Brain); 2003.

[7] Thomson $\mathrm{WM}^{1}$, Poulton R, Broadbent JM, Moffitt TE, Caspi A, Beck JD, Welch D, Hancox RJ. " Cannabis smoking and periodontal disease among young adults"; JAMA. 2008 Feb 6;299(5):525-31. doi: 10.1001/jama.299.5.525.

[8] Hashibe $\mathrm{M}^{1}$, Straif K, Tashkin DP, Morgenstern H, Greenland S, Zhang ZF.; "Epidemiologic review of marijuana use and cancer risk"; Alcohol. 2005 Apr;35(3):265-75.

[9] Kumar RN, Chambers WA, pertwee RG. Pharmacological actions and theraupetic use of cannabis Anaesthesia 2001.

[10] Darling MR, Arendorf TM. Review of the effect of cannabis smoking on oral health. int dent j 1992.

[11] Pacifici R, Zuccaro p, Pichini s, et al. Modulation of the immune system in cannabis users. JAMA 2003.

[12] Momen-Heravi F, Kang P.; "Management of cannabis-induced periodontitis via resective surgical therapy: A clinical report"; J Am Dent Assoc. 2017 Mar;148(3):179-184. doi: 10.1016/j.adaj.2016.10.009. Epub 2016 Nov 18.

[13] Haroutounian S, Ratz Y, Ginosar Y, Furmanov K, Saifi F, Meidan R, Davidson E.; "The Effect of Medicinal Cannabis on Pain and Quality-of-Life Outcomes in Chronic Pain: A Prospective Open-label Study.”; Clin J Pain. 2016 Dec;32(12):1036-1043. 